\title{
Application of Computer Feedback Technology Software in the Rehabilitation of Motor Function
}

\author{
Sheng Guo \\ P. E. College, Beihua University \\ Jilin City, Jilin Province, China
}

Keywords: Feedback, Motor function, Sensing information, Rehabilitation.

\begin{abstract}
Feedback is an important way of information transmission in motor function output. Movement-related in-formation can be transmitted as proprioceptive, haptic, visual and/or audial information to the central nervous system for control and modification of the temporal and spatial precision of motor output. Here presented is a systematic discussion on the principle, technique and effect for some typical motor function rehabilitation methods such as treatment based on EMG feedback, robot-aided movement therapy, high-level perceptional feedback and motor training effect with 3-D virtual reality environment.
\end{abstract}

\section{Introduction}

The normal motor functions are under the domination and control of the nervous system, on the one hand, the active control process controls the process from central motor nerve, peripheral motor nerve to muscles, and on the other hand, the information feedback process controls the process from sensory organ, sensory nerve to sensorium. Tactile information, visual information, auditory and other somatosensory information will regulate the implementation of the motor function in a bid to ensure the accurate completion of the motor functions. Stroke and other cardiovascular and cerebrovascular diseases, traumatic brain injury, spinal cord injury and peripheral nervous system function lesions will affect patients' normal motor functions, and various types of rehabilitation therapy and rehabilitation training measures will help the recovery of the motor functions to varying degrees. In recent years, neurophysiology, neuropsychology, behavioral science and clinical research results show that information feedbacks relevant to the motor function execution from the internal human bodies and external parts have a significant impact on motor function rehabilitation training and rehabilitation treatment effect. The article sums up the application of several main computer feedback technologies, such as EMG signal feedbacks, robot auxiliary motor rehabilitation trainings, higher cognitive processes and three-dimensional virtual technology, in rehabilitation treatment of the motor functions from mechanism, realizing methods, treatment effect and other aspects of feedbacks.

\section{Impact of sensory information feedback on the execution of the motor function}

Realization of human motor functions contains a complex process of neural activities, whether reflection-typed knee-jerk reflection or autonomous breathing and digesting motions, whether active running motion or passive posture balanced control, all contain both motor nerve excitation and conductive processes and a complex sensory nerve activating process. Studies have shown that the control of motor function contains relatively independent nervous structures involved in the control of different functions. Information feedbacks of the sensory nerves have an important impact on accuracy and precision of motor function completion and damages on sensory nerve pathways will affect motor functions significantly. Proprioception dominates main channels to acquire feedback information, including force, velocity, and displacement. Proprioceptive information comes from the 
proprioceptor of the skin, muscles, joints and other parts, which sense motion, muscular tension, balance and strength parameters under the static or dynamic states in order to sense relative motions between different parts in the internal body or the mass motion of the body, so the proprioceptive information plays an important role in completing the motor functions. At the same time, the visual and auditory feedback has an obvious influence on the execution of the motor functions; in particular, the visual feedback acts a big role in guaranteeing the stability and accuracy of executing the motion functions. In addition, the high-level cognitive processes of the nervous system and psychological effects have significant impacts on realizing the motion functions. Therefore, the damage of the motor function is not only related to the motor nerve but also the sensory nerve information feedback, which has a major impact on the rehabilitation treatment effect of the motion functions.

\section{Computer feedback technology in motor function rehabilitation treatment}

Neural network reconstruction based on EMG biofeedback. For most patients with hemiplegia, hemiplegic limb has not lost motor functions completely, and some weak EMG information still exists. EMG biofeedback is used to detect these remnants of the weak EMG, and combine with functional electrical stimulation; and give an electrical stimulation to the patient when the EMG (electromyography) signals generated in the course of the patient's spontaneous activities amount to a certain level (the numeric value to induce the electrical stimulation), causing a muscle contraction with functional activities as a reward to pay the patient's endeavor. The effective mode of repetitive feedback plus stimulation induces activities left in the lesion part under random controls better, thereby activating proprioceptor and skin sensory input, restoring and building proprioceptive feedback pathway to reach the reconstruction of the neuromuscular functions. In the field of neurophysiology, nerve germination, a unique physiological phenomenon in the neural system is used to create a new neural pathway by generating new neural axons, dendrites and synapses, build new neural connections to replace damaged neural signaling pathway, making the reconstruction of the neural network possible. The Equipment utilizing the principle in the research has been widely used in the clinical area. The equipment adjusts the appropriate point to stimulus intensity by detecting and analyzing the weak EMG signals issued by the patients in need of motions; and the patient will reach and exceed the induced point intensity through contraction of their own muscles to gain the electrical stimulation. As soon as the patients increase their EMG index successfully, the equipment will turn up the inducing point intensity automatically, promoting the patients to increase muscle contraction and leading to a further electrical stimulation as a result. Controlled clinical studies have shown that the equipment has significant curative effects on improving motion functions of hemiplegic patients' upper limbs, ankle dorsiflexion obstacles, stretching wrist dysfunction, hemiplegic patients’ daily living skills and other aspects.

Robot auxiliary motor function rehabilitation treatment. Studies have shown that long-term exercise activity can not only affect the function of the muscle, but also enhance the spinal motor neurons and neural activities in regions related to the brain. Animal experiments have shown that exercise activities have a great impact on spinal motor neurons, wherein long-term physical exercises can prevent the recession of the spinal motor neurons caused by age. This is the foundation of robot auxiliary motor function rehabilitation treatment. And the basic principle lies in driving the patients' limbs to complete some specific motions repeatedly by the mechanical motion device, aiming at improving the motion level of the hemiplegic limbs.

A treatment method called as LOKOMT helps patients generate a motion model similar to walking and the patients' lower limbs are drawn by the robot device to walk passively. In the course of completing passive movement of the patients' legs, the patients' own spinal neural network controlling their gaits will strengthen its activities, and may also encourage the patients to practice their lost neural response function related to walking mentally. Comparison of experimental results indicates that the passive walking trainings or alternative leg movements with limbs driven by the auxiliary mechanical device can improve the level of the lower limbs muscle motion of the patients with severely damaged spinal cords. However, passive trainings have no significant effect on the walking function recovery of the patients with completely damaged spinal cords. Compared with the 
control group receiving conventional rehabilitation treatment, patients with partly damaged spinal cords and stroke get obvious improvements in terms of lower limbs' walking movement abilities after their robot auxiliary walking trainings. Research on the robot auxiliary upper limbs motor function training method has been done at the Massachusetts Institute of Technology (MIT) and other labs. And the method has made a significant treatment effect in motion rehabilitation training on patients with dead brains, even including those patients with severely damaged motor functions. Scheidt and other researches also show that the dynamic and kinematic characteristics of the patients' upper limb movement have been recovered well due to the robot auxiliary training. The results of these studies have proved the effectiveness of the robot auxiliary motor function rehabilitation treatment. Despite its inherent mechanism of action needs further study, no hinder will block the way that the method will become the new development direction of motor function rehabilitation treatments.

Application of higher cognitive methods in the rehabilitation of the motor function. In the learning process of motions, higher cognitive activities of brains are also included, besides the participation of the motor nerve and sensory nerve. This also calls in some motor function rehabilitation treatment methods based on higher cognitive processes, including the imagining movement. Shoham and other experts find that the motor cortex exciting area induced by the imagining movement will be excited after the hemiplegic limb completes the motor function in practice, and the imaging movement will accelerate the learning of motor techniques or the recovery of motor function. The motion and relearning method is a widely used motor function rehabilitation treatment. The method regards the rehabilitation training of the motor function after the central nervous system is damaged as a course combining relearning with retraining, wherein the imagining movement plays an important role in training contents. The method is based on neurophysiology, sports science, biomechanics, behavioral science and other theories and reeducates patients in accordance with scientific motor learning methods in a move to recover their motor functions under the precondition of emphasizing subjective participation of the patients and importance of cognition. The curative effect of the motor and relearning method has been confirmed in terms of motor function recovery of the hemiplegic patients' upper limbs and other aspects, and applied to clinic motor function rehabilitation training. Mandatory motor function rehabilitation treatment is another motor function rehabilitation treatment method. The method strengthens exercise activities of terrible-damaged limbs through limitation of limbs activities less-damaged or contralateral, aiming at regaining the motor functions of the limbs. From neuropsychology's point of view, CIMT gives a hand to patients to overcome the view of learned non-use in their minds by means of some certain mandatory regulatory measures. And CIMT improves the motor function of the hemiplegic limbs via higher cognitive activities, including psychological feedback. An abundance of research results demonstrate that CIMT treatment method will lead to recovery of many neurons close to the damaged cortex dominated area, which represents the dominated excitatory balanced rotation of muscle activities between the bilateral cerebral hemispheres. CIMT has got wide application and research in clinical practice and made a significant treatment effect in terms of motor function dysfunction and other aspects.

Application of 3D virtual technology in the recovery of the motor function. As a new technology, virtual reality (VR) technology provides users with virtual scenes and virtual objects and offers users a way to interoperate or make dialogues with virtual objects. The technology is generated by combination of computer graphics technology and sensor control technology. The technology is mainly featured with interactive operating environment provided for the operating user, in particular, real-time and realistic visual, auditory, tactile and other feedback information. At present, the technology is on the way to apply the combination of visual and other sensory information and virtual technology for motor function recovery. Jack has studied a virtual system available for hemiplegic patients with stroke to get the motor rehabilitation training by means of data glove to supply tactile feedback information and the patients have achieved remarkable rehabilitation training effect in 2001. Merians tests the effect of virtual realistic technology on the motor function rehabilitation training at the chronic recovery phase of stroke patients and the result shows that the 
level of daily activities recovery will get improved on the condition that VR is in use to strength feedback information in the course of standard motor function recovery training. VR technology has many unique advantages. First, it allows the trainer in the absence of any dangerous conditions to simulate and complete some virtual activities, allowing the treating physician to adjust the virtual environment parameters on the basis of individual condition in order to get a better rehabilitation effect. At the same time, the VR technology is available to exercise rehabilitation training, regulating and controlling the feedback information inside and outside of the body, including the scene information, strength and size. In addition, patients' passions to join in and their training effects will be stimulated due to more vivid and realistic virtual environment provided by VR. Researched results show that VR has no obvious effect on the motor function training of healthy people; as a result, curative effect of VR on patients with motor dysfunction may be due to increased passion of patients to new VR training environment and the patients' subjective psychological factors. However, as far as some patients concerned, the individual's subjective psychological effect, such as passion in participation, is just of particular importance. Nevertheless, VR treatment may cause some negative effects in the event of neglecting some important personal factors. In particular, an obvious delay of some image information reflecting motion behavior will have great influences on VR training treatment effect because the delay will result in patients' feelings similar to seasickness.

\section{Conclusions}

The model for the modern database is mostly relation model, whose essence is the database structure model expanded from e-r model . Semantic model is put forward in 1988 that is based on the semantic objects and properties, the building structure of which is similar to the OOP method. As the chart shows is the semantic model database views:

\section{References}

[1] Dario GL, Aron SB, Ian MF. Enhancement of motor rehabilita-tion through the use of information technologies. Clinical Biome-chanics, 2006; 21(2) : 8

[2] Yoram B, Ariel M. Auditory feedback control for improvement of gait in patients with Multiple Sclerosis. Journal of the Neurological Sciences, 2007; 254(1-2) : 90

[3] Jeffrey MS, Carl WL, Louis P,et al.Discrete bandwidth visual feedback increases structure of output as compared to continuous visual feedback in isometric force control tasks. Clinical Biomechanics, 2006; 21(10) : 1042

[4] Han R, Ni CM. Effect of electromygraphic biofeedback on upper extremity function in patients with hemiplegia after stroke. Chin J Rehabil Theory Practice,2005;11(3)

[5] Liu YH, Zhang SZ, Ge RL. The clinical observation on the treat-ment of ankle disorder of paralysis with EMG feedback. Chinese Journal of Rehabilitation Medicine, 2005; 20(5) $: 379$ 